\title{
Sensitivity and specificity of cerebrospinal fluid glucose measurement by an amperometric glucometer
}

\author{
Ahmed S. Alkhalifah, MD, Khalid A. Alqatari, MD, Abdullah A. Alkhalifa, MD, Baneen A. Akakah, MD, Zainab S. Alobaid, MD.
}

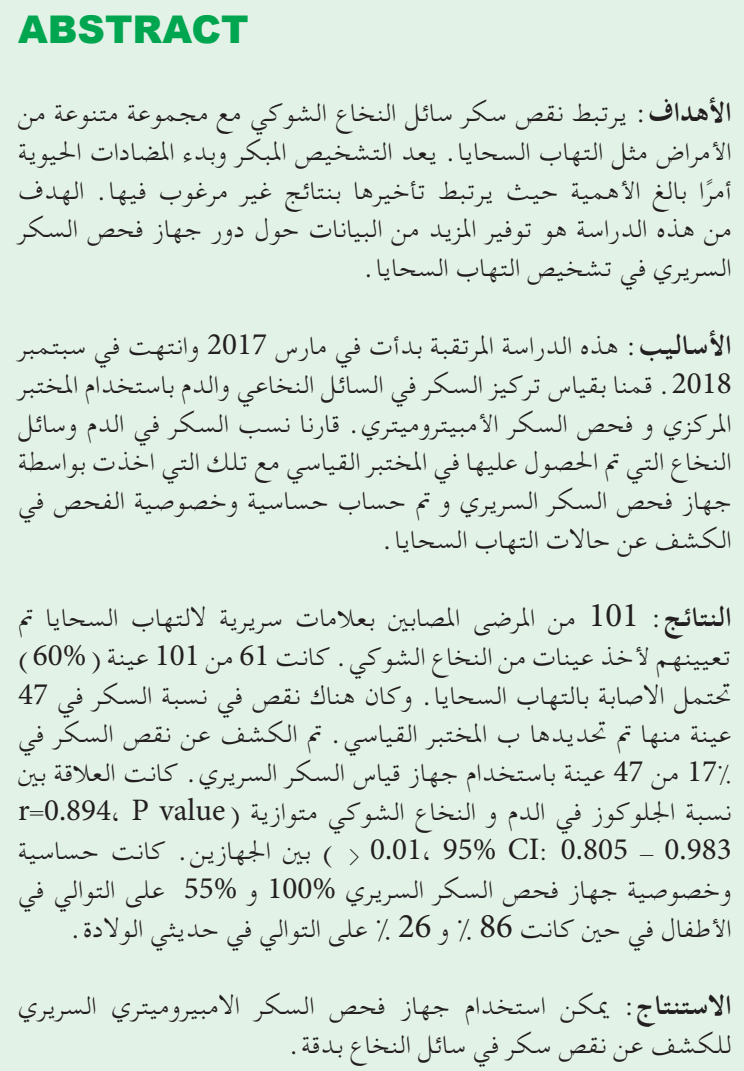

Objectives: To provide more data regarding the role of an amperometric glucometer in diagnosing meningitis.

Methods: This is a prospective study conducted at the Pediatric and Neonatology Department, Qatif Central Hospital, Qatif, Saudi Arabia between March 2017 and September 2018. We measured glucose concentrations in cerebrospinal fluid (CSF) and blood using a central laboratory and amperometric glucometer (AG). We compared CSF/blood glucose ratios obtained in a central laboratory from clinical bedside examination with a glucometer, and calculated the sensitivity and specificity for detecting cases of meningitis.
Results: A total of 101 patients with clinical suspicion of meningitis were recruited for CSF sampling. Of 101 CSF samples, 61 (60\%) were suggestive of meningitis. Of 101 samples, 47 had hypoglycorrhachia identified by a standard laboratory, and $17 \%$ of them were also detected by AG. The correlation between CSF/blood glucose by AG and laboratory ratios was substantial ( $\mathrm{r}=0.894, p<0.01,95 \% \mathrm{CI}: 0.805-0.983)$. The AG sensitivity was $100 \%$ and specificity was $55 \%$ in pediatric cases, while in neonates the sensitivity was $86 \%$ and the specificity was $26 \%$.

Conclusion: Amperometric glucometers can be used to detect hypoglycorrhachia accurately. This pointof-care testing tool is easily accessible and can be used by health care providers for cases suspected of meningitis.

Keywords: cerebrospinal fluid, glucose, point-of-care testing, chemistry, analytic, meningitis

Saudi Med J 2020; Vol. 41 (6): 602-606 doi: 10.15537/smj.2020.6.25118

From the Department of Pediatrics, Qatif Central Hospital, Al Qatif, Kingdom of Saudi Arabia.

Received 5th March 2020. Accepted 13th May 2020.

Address correspondence and reprint request to: Dr. Ahmed S. Alkhalifah, Department of Pediatrics, Qatif Central Hospital, Al Qatif, Kingdom of Saudi Arabia. E-mail:Ahmedsk2@gmail.com ORCID ID: https://orcid.org/0000-0001-9277-8511

$\mathrm{H}$ ypoglycorrhachia (abnormally low cerebrospinal fluid (CSF) glucose) is associated with a variety of diseases that can affect both neonates and pediatric age groups, such as meningitis, glucose transporter 1 deficiency, central nervous system leukemia, and others. ${ }^{1,2}$ One of the most common diseases that causes hypoglycorrhachia is bacterial meningitis, which has high mortality and morbidity rates and accounts 
for more than 1.2 million cases estimated to occur worldwide each year. ${ }^{3}$ The International Journal of Infectious Diseases published an article in 2012 stating that there were 274 reported cases of meningococcal meningitis in Saudi Arabia in 2001. ${ }^{4}$

Early diagnosis and initiation of meningitis management are of utmost importance inform of decreasing mortality and improving outcomes significantly..$^{5}$ In 2016, Bodilsen et $\mathrm{al}^{6}$ observed that the delay in the management of bacterial meningitis more than 6 hours from admission was associated with undesirable outcomes compared to those who received the treatment within 2 hours. Immediate bedside diagnosis of meningitis and initiation of treatment, like cytological analysis is crucial, though not feasible in many situations. Other measures could improve the decision of starting antibiotics earlier and improve outcomes. Evidence for such methods, including pointof-care (POC) strip reagent testing for cerebrospinal fluid (CSF) glucose, protein, and leucocytes, are lacking, though it could facilitate decision-making if demonstrated to be accurate. Several studies have analyzed other methods, which have been suggested using a POC glucometer to determine glucose level in $\mathrm{CSF}^{7,8}$ In 2015, Nirupam et al ${ }^{7}$ evaluated the accuracy between an amperometric glucometer (AG) and an autoanalyzer (AA) with 50 neonates and concluded that $A G$ is a potential tool for bedside measurement of CSF glucose. Another study with pediatric subject up to the age of 12 years found that glucometer strips have $87 \%$ sensitivity and $96 \%$ specificity. ${ }^{8}$ However, both studies had a limited sample size.

In this study, we aim to provide more data regarding the role of AG in facilitating the detection of suspected meningitis in clinical practice.

Methods. This study was conducted in the Pediatric and Neonatology Department, Qatif Central Hospital $(\mathrm{QCH})$, Qatif, Saudi Arabia. The prospective study period lasted for 15 months between March 2017 and September 2018. Ethical approval was obtained by an institutional review board from $\mathrm{QCH}$.

Pediatric patients from 0 to 14 years who were admitted to $\mathrm{QCH}$ with a clinical suspicion of meningitis were included in the study. Preterm neonates, patients with missing data or delay in sample analysis were

Disclosure. Authors have no conflict of interests, and the work was not supported or funded by any drug company. excluded from the study. A total of 101 CSF samples were obtained from neonates and pediatric patients. All CSF sampling was performed by pediatric residents using a lumbar puncture. Cerebrospinal fluid glucose was tested by AG. Cerebrospinal fluid samples were sent immediately for processing in the laboratory for cytology (white blood cell and red blood cell count), cultures, gram staining, latex agglutination testing, and biochemical analysis. Biochemical analysis was carried out with the Siemens Dimension Glucose analysis device (Siemens Healthcare GmbH., Erlangen, Germany). Sampling and laboratory analysis were carried out within a window of 30-60 minutes. We excluded patients with delayed CSF laboratory analysis of more than one hour from the study.

Bedside measurement was performed by pediatric residents immediately after collection. The glucometer used in this study was the FreeStyle Optium Neo Rev. A 05/14 (Abbott's Diabetes Care, Berkshire, UK) with an amperometric system that measures the electric current generated at a specific point in time by the glucose reaction. Serum blood glucose was measured within one hour before CSF sampling.

Patients were classified into the normal CSF group or high clinical suspicion of meningitis group according to CSF analysis. The cases identified were thought to have high suspicion of meningitis by having positive CSF culture, hypoglycorrhachia defined as a CSF-toserum glucose ratio of $<0.6,{ }^{9} \mathrm{CSF}$ protein more than $100 \mathrm{mg} / \mathrm{dL},{ }^{10}$ or CSF white blood cell count more than 6 cells/uL for children older than 3 months of age, more than 9 cells/uL for infants 29 to 90 days, and more than 20 cells/uL for neonates. ${ }^{10-12}$

Statistical analysis. We used IBM SPSS Statistics for Windows, version 25.0 (IBM Corp., Armonk, N.Y., USA) program for statistical analysis. A t-tailed test with a $p$-value of $<0.05$ was used to determine significance. Shapiro-Wilk normality testing was applied for describing the distribution of the data. The concordance correlation coefficient was analyzed, with a score of one indicating perfect agreement.

The primary outcome of the study was to verify AG measurements in comparison with standard laboratory biochemical analysis in children with high suspicion of meningitis. The secondary outcome was evaluating the accuracy of glucose values obtained by AG compared with standardized methods used at Qatif Central Hospital.

Results. A total of 150 patients had CSF sampling during the study's duration at $\mathrm{QCH}$. In terms of exclusion, 49 samples were excluded from the study 
as demonstrated in Figure 1. We analyzed 101 patients with clinical suspicion of meningitis in total $(54 \%$ pediatric and $46 \%$ neonates). From each, a CSF sample was taken for CSF glucose measurement. From the 101 patients evaluated, $61 \mathrm{CSF}$ samples (26 pediatric and 35 neonates) indicated high suspicion of meningitis by laboratory analysis. Proportionally, $17 \%$ of CSF samples with high suspicion of meningitis had abnormal white blood cell counts while 21 samples had significantly high protein count. The CSF characteristics are summarized in Table 1.

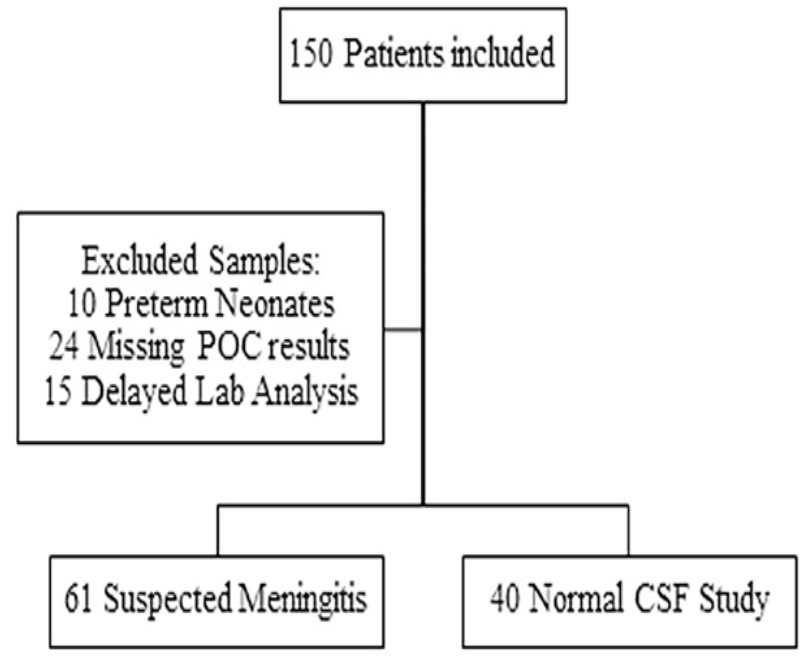

Figure 1 - Flow diagram showing flow of participants. POC: point-ofcare, CSF: cerebrospinal fluid
A total of 47 samples had hypoglycorrhachia identified by the standard laboratory. Proportionally, $17 \%$ of them were also detected by the AG. Most of the remaining 54 cases that were reported to have normal glucose levels by the laboratory were also found to be normal by AG (98\%). The difference in measurements between CSF laboratory glucose and AG values were normally distributed (Figures 2 \& 3) with Shapiro-Wilk normality testing significance of 0.13 .

The correlation between the CSF/blood glucose by $\mathrm{AG}$ and LAB ratios was substantial $(\mathrm{r}=0.894, p<0.01$, 95\% CI: 0.805 - 0.983) (Figure 4). The paired-mean difference for glucose measurements between the 2 methods was $29.11 \pm 19.76 \mathrm{mg} / \mathrm{dl}(1.6 \pm 1.1 \mathrm{mmol} / \mathrm{L})$ $(p<0.000)$ for all CSF samples while in cases of hypoglycorrhachia, the paired-mean difference was not significant $(4.2 \pm 4.9 \mathrm{mg} / \mathrm{dl}[0.2 \pm 0.3 \mathrm{mmol} / \mathrm{L}], p=0.18)$.

When the POC glucometer was used to enhance confirming suspicion of meningitis, the sensitivity of the AG was $100 \%$ and specificity was $55 \%$ in pediatrics, while the sensitivity of neonates was $86 \%$ and specificity

Table 1 - Initial cerebrospinal fluid analysis.

\begin{tabular}{lcc}
\hline Fluid analysis & $\begin{array}{c}\text { Suspected } \\
\text { Meningitis }(\mathbf{n}=61)\end{array}$ & $\begin{array}{c}\text { Normal } \\
(\mathbf{n}=40)\end{array}$ \\
\hline White cell count $(\mathrm{cell} / \mathrm{\mu l})$ & $169.23(0-8000)$ & $2.2(0-17)$ \\
Protein $(\mathrm{g} / \mathrm{l})$ & $87.65(12-336)$ & $41.83(14-89)$ \\
Glucose $(\mathrm{mg} / \mathrm{dl})$ & $59.57(21-131)$ & $73.56(44-138)$ \\
\hline
\end{tabular}

Values are presented as mean (interquartile range)

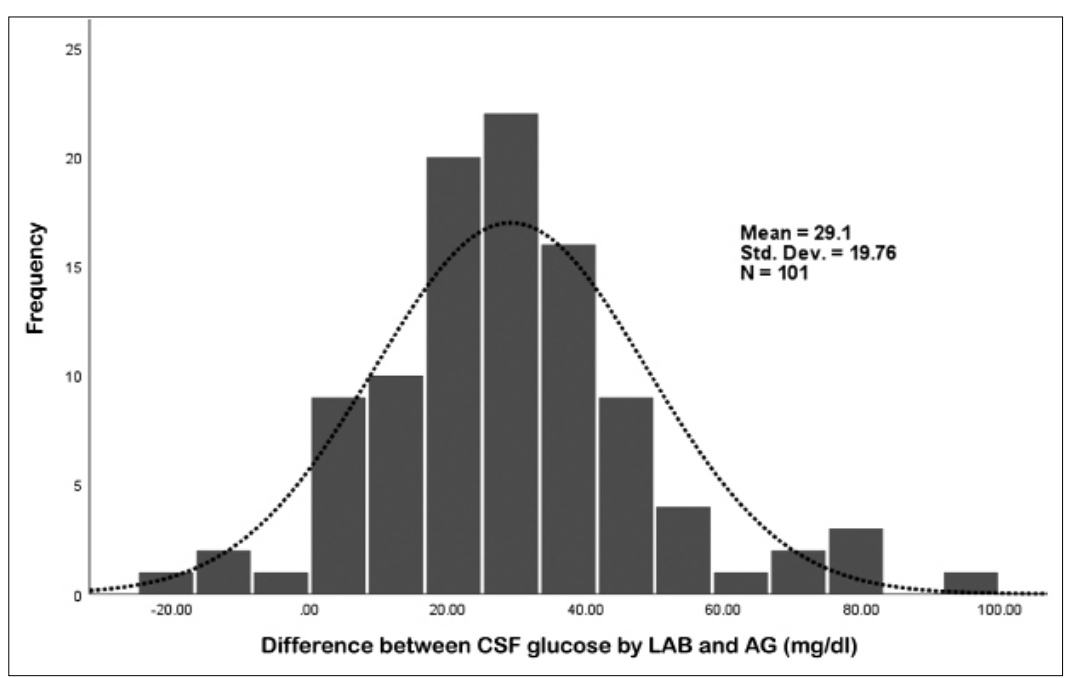

Figure 2 - Histograms for the difference between CSF glucose measurements by LAB and AG. CSF: cerebrospinal fluid, LAB: standard laboratory, AG: amperometric glucometer 


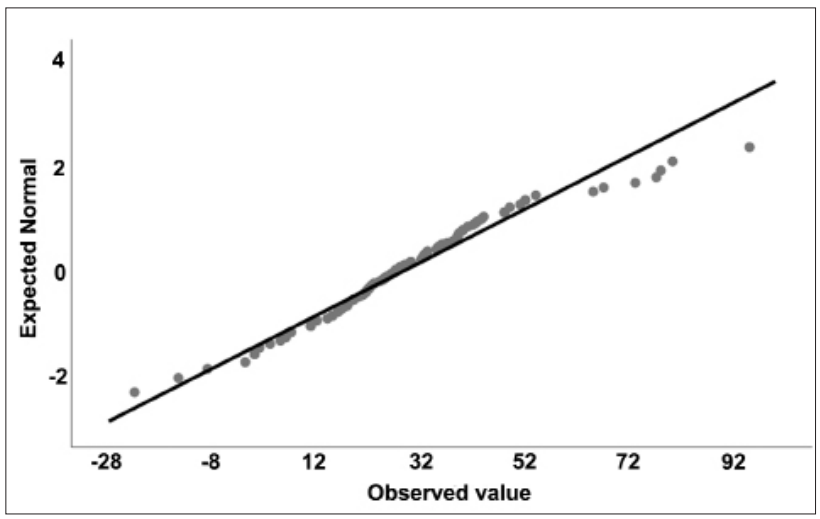

Figure 3 - Q-Q Plot for the difference between CSF glucose measurements by $\mathrm{LAB}$ and $\mathrm{AG}$

was $26 \%$. Both groups combined showed that $A G$ is $89 \%$ sensitive and only $42.3 \%$ specific.

Discussion. Measuring CSF glucose is an important diagnostic tool for a variety of diseases. In cases of meningitis, hypoglycorrhachia is a significant finding. Early detection of hypoglycorrhachia in patients suspected to have meningitis can aid in the early initiation of antibiotics and thus improve mortality and morbidity.

There have been many studies published regarding different POC methods to detect hypoglycorrhachia. ${ }^{7,8,13-16}$ Point-of-care tools and methods have pre-analytical and analytical errors while diagnosis also depends on the health care provider's knowledge surrounding how to use them. In 2018, Mazumder et $\mathrm{al}^{13}$ employed a urine reagent strip test as a quantitative method for CSF analysis of 100 samples and indicated that it is highly specific (100\%), but less sensitive for glucose. This study also quantified leukocytes and protein, endowing the urine strep method with more value in POC CSF analysis. This study and many others indicated that urine reagent strips can be used in detecting pleocytosis and low glucose in CSF at facilities that do not have the appropriate laboratory settings for CSF analysis, though it does not yield numeric readings and cannot be applied to differentiate between bacterial or viral meningitis. ${ }^{15,16}$ Another group used the iSTAT CHEM8 and CG4+ cartridges for analysis with correlating results compared to traditional methods for measuring CSF glucose, ${ }^{14}$ which is also a helpful method for fast detection of hypoglycorrhachia but not as accessible as other POC tools.

Few studies have investigated the ability of POC glucometers to measure CSF glucose, especially in the

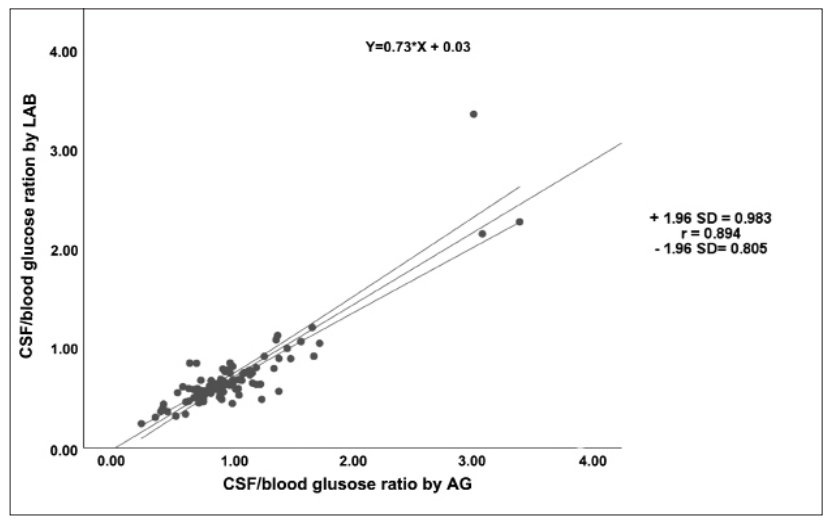

Figure 4 - Correlation curve of the CSF/blood glucose ratio obtained by amperometric glucometer and the laboratory

pediatric age group. ${ }^{7,8}$ Different models of glucometers with various technologies are widely available at lower prices compared to other tools. In 2015, Nirupam et $\mathrm{al}^{7}$ assessed the CSF glucose in 50 neonates, of which 13 had meningitis. They demonstrated the difference between a photometric glucometer and an AG, concluding that a photometric glucometer is poorly correlating with standard laboratory methods compared with an AG, which was significantly correlated with $100 \%$ sensitivity in detecting CSF hypoglycorrhachia in cases with meningitis.?

Rousseau et $\mathrm{a}^{17}$ analyzed 172 CSF samples utilizing a POC AG and standard laboratory methods. The median age of the study population was 55 years (range: 28-77 years). They concluded that a bedside glucometer has a sensitivity of $94.1 \%$ (95\% CI: 71.3-99.9\%) and specificity of $91 \%$ (95\% CI: 85.3-95\%). ${ }^{17}$

This study included a larger sample size (101 CSF samples of the neonatal and pediatric age groups) compared to other studies of the same age group. ${ }^{7,8}$ Using AG for CSF glucose measurement, the study found that the readings were correlated significantly with laboratory results. The paired-mean difference for AG CSF glucose measurements was $29.11 \pm 19.76 \mathrm{mg} / \mathrm{dl}$ $(1.6 \pm 1.1 \mathrm{mmol} / \mathrm{L})$ compared with laboratory methods across all CSF samples, whereas the paired-mean difference was $4.2 \pm 4.9 \mathrm{mg} / \mathrm{dl}(0.2 \pm 0.3 \mathrm{mmol} / \mathrm{L})$ when compared for cases with hypoglycorrhachia. This narrow range difference is acceptable for clinical use and supports the findings described by Nirupam et al in 2015.7

Overall, this study showed that AG is highly sensitive in detecting hypoglycorrhachia, but has low specificity (sensitivity: $89 \%$; specificity: $42.3 \%$ ) compared to what was reported by Rousseau et a ${ }^{17}$ when they performed a 
similar study on an older age group (sensitivity: $94.1 \%$; specificity: 91\%).

Study limitations. Few cases were discovered to have hypoglycorrhachia, which can affect the sensitivity and specificity of AG, as well as the time difference between sampling, which can be minimized further in facilities where the laboratory method can be applied at the same time as AG to CSF samples. Studies with larger sample size can overcome these limitations.

In conclusion, AG can be a potential tool for measuring CSF glucose for the rapid detection of hypoglycorrhachia with just slight overestimation in glucose readings. This overestimation can be explained by the time difference between measurements by the 2 methods as described by Rajesh et $\mathrm{a}^{18}$ in 2010 when they established that delays in CSF analysis can decrease glucose value.

Acknowledgment: We would like to thank Cambridge Proofreading LLC (www.proofreading.org) for English language editing.

\section{References}

1. Silver TS, Todd JK. Hypoglycorrhachia in pediatric patients. Pediatrics 1976; 58: 67-71.

2. Klepper J, Leiendecker B. GLUT1 deficiency syndrome-2007 update. Dev Med Child Neurol 2007; 49: 707-716.

3. Epidemiology of Meningitis Caused by Neisseria meningitidis, Streptococcus pneumoniae, and Haemophilus influenzae, 2016 [Internet]. Atlanta (GA): Centers for Disease Control and Prevention; 2016.

4. Ceyhan M, Anis S, Htun-Myint L, Pawinski R, SorianoGabarró M, Vyse A. Meningococcal disease in the Middle East and North Africa: an important public health consideration that requires further attention. Int J Infect Dis 2012; 16: e574-e582.

5. Sheley J, Willman D, Downen J, Bergman S. Investigation of the selection and timing of pharmacological therapy in community-acquired bacterial meningitis. $P$ T 2016; 41: 437-441.
6. Bodilsen J, Dalager-Pedersen M, Schønheyder HC, Nielsen H. Time to antibiotic therapy and outcome in bacterial meningitis: a Danish population-based cohort study. BMC Infect Dis 2016; 16: 392.

7. Nirupam N, Nangia S, Saili A. Neonatal cerebrospinal fluid glucose estimation using glucometers. The Journal of NearPatient Testing \& Technology 2015; 14: 88-91.

8. Akram DS, Shaikh AM. Determination of cerebrospinal fluid sugar by glucometer. J Pak Med Assoc 2002; 52: 422-423.

9. Nigrovic LE, Kimia AA, Shah SS, Neuman MI. Relationship between cerebrospinal fluid glucose and serum glucose. $N$ Engl J Med 2012; 366: 5766-578.

10. Kim KS. Bacterial meningitis beyond the neonatal period. In: Cherry JD, Harrison GJ, Kaplan SL, et al., editors. Feigin and Cherry's Textbook of Pediatric Infectious Diseases, 7 th. Philadelphia (PA): Elsevier Saunders; 2014. p. 425.

11. Akpede O, Abiodun PO, Sykes M, Salami CE. Childhood bacterial meningitis beyond the neonatal period in southern Nigeria: changes in organisms/antibiotic susceptibility. East Afr Med J 1994; 71: 14-20.

12. Byington CL, Kendrick J, Sheng X. Normative cerebrospinal fluid profiles in febrile infants. J Pediatr 2011; 158: 130-134.

13. Mazumder S, Ramya BS, Biligi DS. Utility of urine reagent strips in cerebrospinal fluid analysis: an aid to bedside diagnosis of meningitis. Indian J Pathol Microbiol 2018; 61: 356-359.

14. Lefrere B, Plantamura J, Renard C, Ceppa F, Delacour H. Biochemical analysis of cerebrospinal fluid in the laboratories of deployed medical treatment facilities: are Multistix 10 SG strip and iSTAT useful? J R Army Med Corps 2017; 163: 397-400.

15. Heckmann JG, Engelhardt A, Druschky A, Mück-Weymann M, Neundörfer B. Urine test strips for cerebrospinal fluid diagnosis of bacterial meningitis. Med Klin (Munich) 1996; 91: 766-768. German

16. Bortcosh W, Siedner M, Carroll RW. Utility of the urine reagent strip leucocyte esterase assay for the diagnosis of meningitis in resource-limited settings: meta-analysis. Trop Med Int Health 2017; 22: 1072-1080.

17. Rousseau G, Asmolov R, Grammatico-Guillon L, Auvet A, Laribi S, Garot D, et al. Rapid detection of bacterial meningitis using a point-of-care glucometer. Eur J Emerg Med 2019; 26: 41-46.

18. Rajesh NT, Dutta S, Prasad R, Narang A. Effect of delay in analysis on neonatal cerebrospinal fluid parameters. Arch Dis Child Fetal Neonatal Ed 2010; 95: F25-F29. 